REVUE DE L'INSTITUT

FRANC CAIS D'HISTOIRE

EN ALLEMAGNE

\section{Revue de l'IFHA}

Revue de l'Institut français d'histoire en Allemagne

$6 \mid 2014$

IFHA 6

\title{
Rück/Blick : un cycle de manifestations consacré par l'IFHA au centenaire des débuts de la Première Guerre mondiale
}

\section{Pierre Monnet}

\section{OpenEdition}

\section{Journals}

Édition électronique

URL : http://journals.openedition.org/ifha/7996

DOI : $10.4000 /$ ifha. 7996

ISSN : 2198-8943

\section{Éditeur}

IFRA - Institut franco-allemand (sciences historiques et sociales)

\section{Édition imprimée}

Date de publication : 31 décembre 2014

ISSN : 2190-0078

\section{Référence électronique}

Pierre Monnet, «Rück/Blick: un cycle de manifestations consacré par l'IFHA au centenaire des débuts de la Première Guerre mondiale », Revue de l'IFHA [En ligne], 6 | 2014, mis en ligne le 22 décembre 2014, consulté le 01 mai 2019. URL : http://journals.openedition.org/ifha/7996 ; DOI : 10.4000/ ifha.7996

Ce document a été généré automatiquement le 1 mai 2019.

(CIFHA 


\title{
Rück/Blick : un cycle de manifestations consacré par l'IFHA au centenaire des débuts de la Première Guerre mondiale
}

\author{
Pierre Monnet
}

1 L'année 2014 a été pour l'IFHA marquée entre autres par le souci de mettre en perspective le centenaire de la déclaration de la Première Guerre mondiale en 1914. Ce conflit a bouleversé l'ensemble du monde et du siècle, mais aussi la science et les historiens. Afin de porter cent ans plus tard un regard critique et partagé sur l'événement (d'où le choix du titre général allemand Rück/Blick coupé en deux pour signifier à la fois le regard rétrospectif et l'observation naviguant entre le passé et le présent), et au moment où l'université Goethe, lieu d'ancrage de l'IFHA, célébrait le centenaire de sa propre fondation (en octobre 1914), l'institut a décidé de mettre en place ou de soutenir un cycle de manifestations organisé en liaison étroite avec de nombreux partenaires culturels et scientifiques, et conçu pour varier les supports et les formes afin de toucher un public large et divers: colloques, conférences, exposition, films, débats, lecture, atelier scientifique et pédagogique. Ainsi notre centre a-t-il souhaité pouvoir contribuer à éclairer, dans une approche comparée et interculturelle, cette catastrophe originelle du XXe siècle dont l'Allemagne et la France, parmi tant d'autres, sont sorties à jamais transformées.

2 On trouvera dans les pages qui suivent le détail de plusieurs des temps forts de cet ensemble de manifestations. On en redonne simplement ci-dessous la liste complète afin que chacun puisse juger de l'éventail des formats et rendez-vous proposés par l'IFHA, toujours en lien, en soutien ou en coopération étroite avec ses alliés et associés culturels et scientifiques à Francfort et dans sa région.

3 Le cycle de ces manifestations organisées, co-organisées ou soutenues par l'IFHA a reçu le label officiel de la Mission du centenaire et bénéficié du soutien de l'Institut Français par le biais du fonds d'Alembert. 
Le 7 mai 2014 a été organisée au Hessisches Literaturforum im Mousonturm de Francfort une lecture avec Éric Vuillard de son roman La bataille d'Occident (Arles : Actes Sud, 2012; en allemand: Ballade vom Abendland, Berlin : Matthes \& Seitz, 2014). Le 14 mai 2014 une conférence et un débat ont eu lieu à l'université de Francfort sur le thème « Der Erste Weltkrieg in der deutschen und französischen Erinnerung/La Première Guerre mondiale et les sociétés française et allemande au XXe siècle : interprétations, souvenirs, mémoires ». Cette rencontre a été organisée par l'IFHA et la Société FrancoAllemande de Francfort, avec Gerd krumeich (professeur émérite à l'université de Düsseldorf) et Antoine Prost (professeur émérite à l'université de Paris I PanthéonSorbonne, président du conseil scientifique de la Mission du centenaire à Paris).

Du 22 au 24 mai 2014 un colloque international intitulé «Europa 1914 - der Weg ins Unbekannte " a été organisé à l'université de Francfort par le séminaire d'histoire de l'université Goethe (Christoph cornelißen) avec le soutien de l'IFHA sur la question de l'entrée dans la guerre en 1914, de l'inéluctabilité ou au contraire de l'évitabilité du conflit, entre jeux d'alliance, part des hommes, rôle des structures et des cultures. Avec la participation, entre autres, de Christopher Clark, auteur de Les somnambules, Paris: Flammarion, 2013 (en allemand: Die Schlafwandler: Wie Europa in den Ersten Weltkrieg zog, München : Deutsche Verlags-Anstalt, 2013).

7 Les 10, 17 et 24 juin 2014, l'IFHA a proposé un cycle de films et de débats avec le Filmmuseum de Francfort : La Grande Illusion de Jean Renoir (1937), Westfront 1918. Vier von der Infanterie de Georg Wilhelm Pabst (1938) et un ensemble de documentaires d'époque sur la guerre sous le titre Der Erste Weltkrieg im Filmarchiv. Das European Film Gateway 1914.

Du 11 septembre 2014 au 15 février 2015 une exposition est proposée par le historisches museum de Francfort: "Gefangene Bilder. Wissenschaft und Propaganda im Ersten Weltkrieg ». Cette exposition, dont la conception et le programme d'accompagnement ont associé étroitement l'IFHA et l'Institut Frobenius d'ethnologie de l'université de Francfort, de même que la rédaction du catalogue correspondant (Gefangene Bilder. Wissenschaft und Propaganda im Ersten Weltkrieg, Petersberg: Michael Imhof, 2014), s'est articulée autour d'une série de quinze portraits photographiques de combattants prisonniers issus des colonies françaises internés dans des camps allemands entre 1914 et 1918. Le concept muséographique entend mettre en perspective historique l'implication des colonies dans l'effort de guerre européen, le regard de l'autre sur ces populations " exotiques ", le discours scientifique tenu sur ces soldats et leurs régions d'origines dans un tutoiement délicat et hautement idéologique entre science et propagande.

Du 10 au 12 septembre 2014 s'est tenu au Holzhausenschlösschen de Francfort un colloque international «1914/2014 - Erster Weltkrieg. Kriegskindheit und Kriegsjugend, Literatur, Erinnerungskultur/ 1914/2014 - La Première Guerre mondiale. Enfance et jeunesse en guerre, littérature, cultures de la mémoire", organisé par l'Institut für Jugendbuchforschung (Institut de recherche sur le livre de jeunesse) de l'université Goethe de Francfort (Hans-Heino Ewers) avec le soutien de l'IFHA sur la littérature de jeunesse, la guerre, la propagande à destination des enfants et la mémoire. Dans ce cadre, l'IFHA a organisé une conférence de Stéphane Audoin-Rouzeau (Directeur d'études à l'EHESS, Paris) le 11 septembre par l'IFHA : « Mort des jeunes, mort des étudiants en 1914-1918 ». Le 17 octobre 2014, à la Ziehenschule de Francfort, a eu lieu un atelier scientifique et pédagogique franco-allemand : «Der Erste Weltkrieg im Unterricht in Deutschland und Frankreich: Lehrpläne, Schulbücher, Erinnerungskulturen/Enseigner aujourd'hui la 
Première Guerre mondiale en France et en Allemagne: programmes, manuels, mémoires ». Cette rencontre a été organisée par l'IFHA, avec la Ziehenschule de Francfort, le Schulamt de la Hesse et le soutien de l'Institut Français de Mayence, consacré aux questions de méthode, de didactique, de pédagogie et d'interprétations à travers l'enseignement comparé de la guerre de 1914-1918 entre la France et l'Allemagne.

Enfin, les 24 et 25 octobre 2014 un atelier franco-allemand de jeunes chercheurs a été organisé par l'IFHA à l'université de Francfort : «Den Krieg neu denken ? Neue Quellen und Methoden zur Geschichtsschreibung des Ersten Weltkrieges/ Penser la guerre autrement? Nouvelles sources et méthodes pour écrire l'histoire de la Première Guerre mondiale ». Cette manifestation était consacrée aux nouvelles sources et méthodes issues des tendances historiographiques récentes (histoire culturelle et sociale, nouvelle histoire militaire, etc.) sur la Première Guerre mondiale vue « d'en bas ».

\section{AUTEUR}

\section{PIERRE MONNET}

(EHESS, Paris et IFHA, Francfort-sur-le-Main) 